\title{
One new species and one newly recorded species of the genus Lasiochira Meyrick, 193I (Lepidoptera, Oecophoridae) from China
}

\author{
Aihui Yin', Yanpeng Cai' \\ I Morphological Laboratory, Guizhou University of Traditional Chinese Medicine, Guiyang, 550025, \\ Guizhou, China \\ Corresponding author: Yanpeng Cai (CYP815@hotmail.com)
}

Academic editor: Mark Metz | Received 16 November 2019 | Accepted 6 January 2020 | Published 12 March 2020

http://zoobank.org/7EC6D9A9-34D7-4E01-935A-13F2A3B5A7E9

Citation: Yin A, Cai Y (2020) One new species and one newly recorded species of the genus Lasiochira Meyrick, 1931 (Lepidoptera, Oecophoridae) from China. ZooKeys 918: 143-150. https://doi.org/10.3897/zookeys.918.48544

\begin{abstract}
Lasiochira wuzhishanensis Yin, sp. nov. is described herein from the island province of Hainan, China. It is diagnosed from a similar species, Lasiochira jianfengensis Yin, Wang \& Park, 2014. Both species are sympatric in Hainan province, but the latter is also known in northern Vietnam. Lasiochira pallidiptera Yin, Wang \& Park, 2014 is recorded for the first time from China. Color images of the adults and genitalia of the above three species are provided. In addition, a checklist and a geographical distribution map of all species of Lasiochira Meyrick are included.
\end{abstract}

\section{Keywords}

Checklist, key, morphology, moth, taxonomy

\section{Introduction}

Lasiochira Meyrick, 1931 is a small genus included in the Oecophoridae, with unknown biology. The type species, Lasiochira camaropa Meyrick, 1931, was diagnosed based on two male syntype specimens from Kwanhsien, China. Clarke (1963) designated a lectotype from Meyrick's original material and transferred a second species, L. xanthacma (Meyrick, 1938), from Allotalanta Meyrick, 1913. No further taxonomic work was published on the genus until Wang (2006) treated the Oecophoridae of 
China, including the two known species. Yin et al. (2014) described six new species, but the entire fauna is known only from China, Korea, and Vietnam.

The genus Lasiochira is mainly characterized by having a pale, N-shaped pattern consisting of three joined stripes and several small tufts of erect scales on the forewing (Figs 1-3); the gnathos is often pointed and hooked terminally (Figs 4, 5, 8); the valva usually has dense fine hairs on the distal half (Figs 4, 5, 8); the cornuti are comprised of multiple short, stout spines or plates (Figs 4, 5, 8; arrows); the apophyses posteriores are longer than the apophyses anteriores (Figs 6, 7, 9); the ductus bursae is with sclerotization (Figs 6,7,9); and the signum is transverse, dentate, with a projecting semicircular lobe posteriorly (Figs 6a, 7a, 9) (Yin et al. 2014).

Recent collecting efforts in the Hainan and Hubei provinces produced a new species, described herein, and the first record of L. pallidiptera Yin, Wang \& Park, 2014 in China. The objectives of this study are to describe the new species and update the distributions of the known species.

\section{Material and methods}

All specimens for this study were collected in 2018 from the Hainan and Hubei provinces of China. The descriptive terminology of the anatomical structures follows Wang (2006), Yin et al. (2014) and Kristensen (2003). Photographs of adults were taken using a Canon EOS 6D Mark II camera with an EF $100 \mathrm{~mm}$ f/2.8L MACRO IS USM lens assisted by the EOS Utility 3.10.20 software. Stacked images of the genitalia were captured using a Leica DM4 B upright microscope. Photomontage was performed with the Leica Application Suite X imaging software. Species distribution data were compiled within Microsoft Excel using both published records and specimen label data. The distribution map was produced with the aid of DIVA-GIS 7.5 (Hijmans et al. 2011).

All type specimens are deposited in the Morphological Laboratory, Guizhou University of Traditional Chinese Medicine, Guiyang, Guizhou, China.

\section{Taxonomy}

Lasiochira Meyrick, 1931: 71.

Type species. Lasiochira camaropa Meyrick, 1931, by monotypy.

\section{Checklist of Lasiochira Meyrick species}

1 Lasiochira camaropa Meyrick, 1931: 71

Distribution: China (Sichuan province).

2 Lasiochira flaviterminata Yin, Wang \& Park, 2014: 33

Distribution: China (Chongqing City). 
3 Lasiochira jianfengensis Yin, Wang \& Park, 2014: 25

Distribution: China (Hainan province), Vietnam (North).

4 Lasiochira jiulongshana Yin, Wang \& Park, 2014: 27

Distribution: China (Zhejiang province).

5 Lasiochira pallidiptera Yin, Wang \& Park, 2014: 30

Distribution: China (Hubei province), Korea (Central).

6 Lasiochira rosataenia Yin, Wang \& Park, 2014: 32

Distribution: Vietnam (North).

7 Lasiochira taiwanensis Yin, Wang \& Park, 2014: 31

Distribution: China (Taiwan province).

8 Lasiochira wuzhishanensis Yin, sp. nov.

Distribution: China (Hainan province).

9 Lasiochira xanthacma (Meyrick, 1938: 10)

Distribution: China (Guangdong, Guizhou, Henan, Shaanxi, Shanxi and Yunnan provinces).

\section{Lasiochira wuzhishanensis Yin, sp. nov.}

http://zoobank.org/4001CC9D-E7DA-4374-9BF1-28A607ADAD91

Figs 1, 4, 6, 6a

Material examined. Holotype: $\sigma^{\lambda}$; China: Hainan province, Wuzhishan City, Wuzhishan National Nature Reserve; alt. 650 m; 1854'36"N, 10940'48"E; 10 May 2018; Zhengyong Wang leg.; YC00025. Paratypes: 1 ô, 3 우영 same locality as holotype; alt. $650 \mathrm{~m} ; 18^{\circ} 54^{\prime} 36^{\prime \prime N}, 109^{\circ} 40^{\prime} 48 " \mathrm{E}$; 10-15 May 2018; Zhengyong Wang leg.; $\mathrm{YC} 00026$ o, $\mathrm{YC} 00022$ ㅇ, $\mathrm{YC} 00023$ q, $\mathrm{YAH} 19072$ ㅇ.

Diagnosis. Lasiochira wuzhishanensis Yin, sp. nov. can be distinguished from its congeners by the following two character states: forewing ocherous brown in ground color (Fig. 1); phallus with seven cornuti (Fig. 4, arrows).

Description. Head: Vertex and front white, tinged with pale ocherous yellow on cervical area; labial palpi white, first segment with dark brown scales dorsally, second segment somewhat tinged with pale ocherous brown, denser ventrally, third segment with broad pale ocherous brown ring at distal 1/4; antenna with scape white, covered with pale ocherous yellow scales on dorsal surface, pecten pale yellow, flagellum pale ocherous yellow, ringed with white; proboscis white.

Thorax: Tegula and mesonotum white, with three transverse pale ocherous brown bands; legs pale yellowish white, tibiae and tarsi pale ocherous brown on outer surface and pale ocherous brown and pale yellowish white on inner surface. Forewing (Fig. 1): length 5.5-7.0 $\mathrm{mm}(\mathrm{N}=5)$, about $3.3 \times$ as long as wide, ocherous brown, slightly paler between $\mathrm{CuP}$ and dorsum; an N-shaped pinkish pattern running from basal $2 / 5$ of costa diagonally outward to posterior angle of cell, obliquely to subapical part of costa, enlarged on costal margin, and diagonally narrowed to ventroapical part of termen; innermost stripe edged with two erect scale tufts, tufts black or ocherous brown on inner margin, angle between innermost and middle stripes diffused with blackish brown 

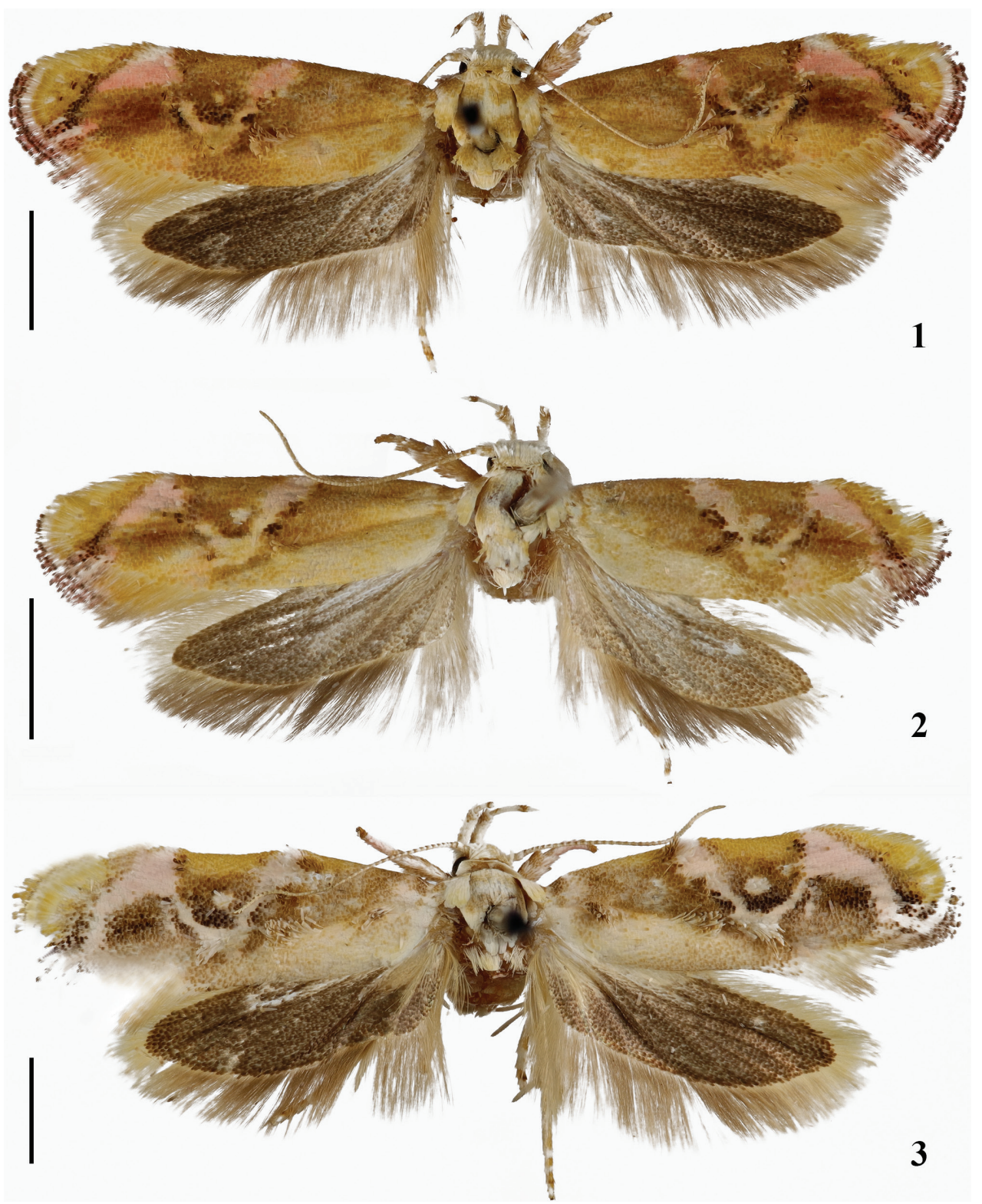

Figures I-3. Adults of Lasiochira species I L. wuzhishanensis Yin, sp. nov., paratype, female, gen. slide no. YAH19072 2 L. jianfengensis Yin, Wang and Park, female, gen. slide no. YC00028 3 L. pallidiptera Yin, Wang and Park, male, gen. slide no. YAH18154. Scale bars: $2.00 \mathrm{~mm}$.

scales, and anteriorly with an erect pale yellowish white scale tuft, middle and outmost stripes both edged with erect scale tufts, tufts black or ocherous brown on outer margins; $\mathrm{CuP}$ with an erect scale tuft near distal $1 / 3$; cilia with basal $3 / 5$ pale ocherous brown, distal 2/5 pinkish white, edged with dark brown; ventral surface grayish brown. Hindwing (Fig. 1): dark gray; cilia yellowish gray. 


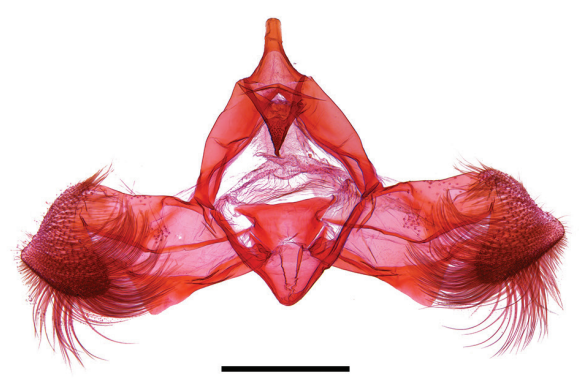

4

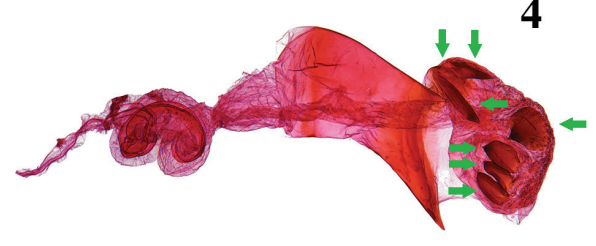

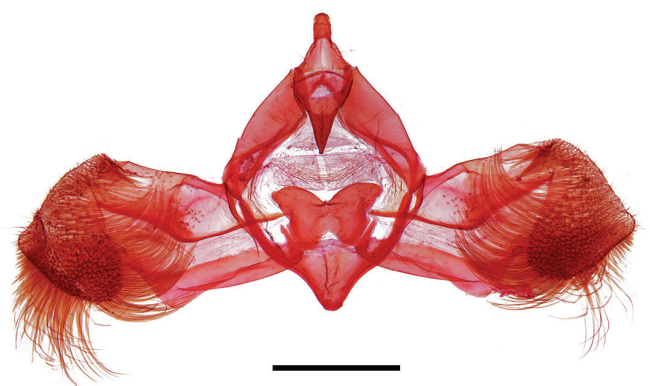

5
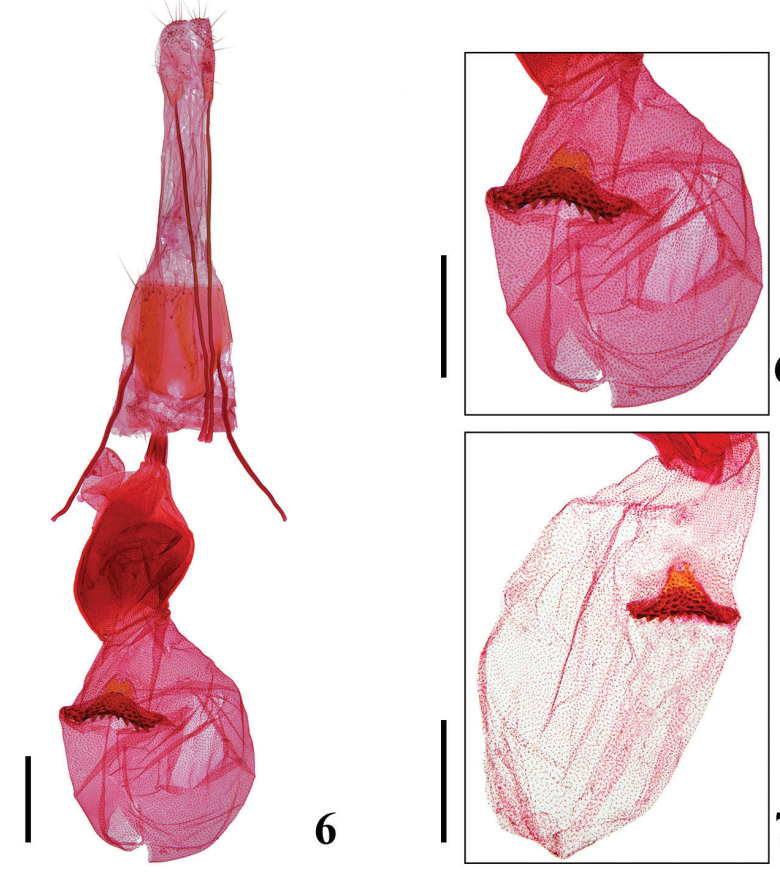

\section{$6 \mathbf{a}$}
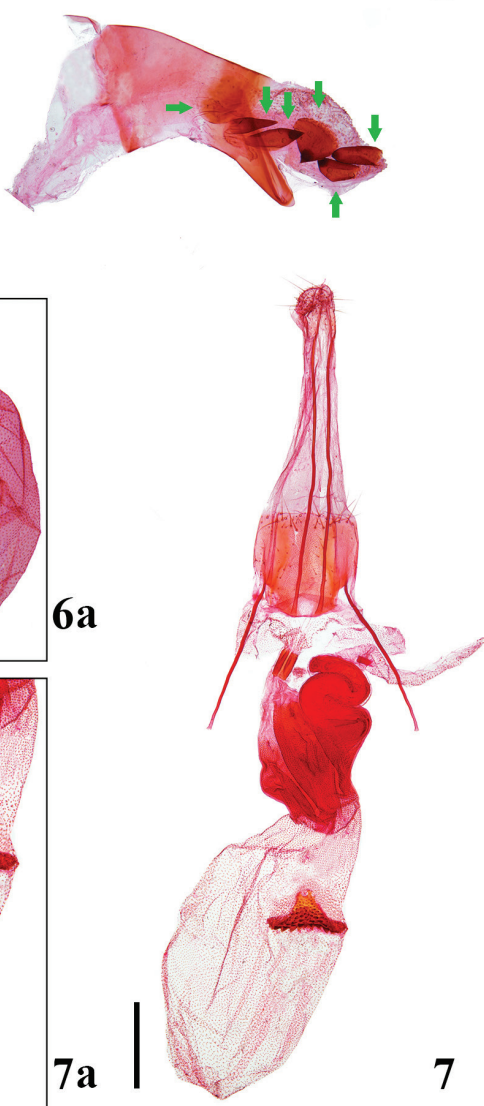

Figures 4-7. Male and female genitalia of Lasiochira species 4 L. wuzhishanensis Yin, sp. nov., holotype, male genital capsule and phallus, gen. slide no. YC00025 5 L. jianfengensis Yin, Wang and Park, male genital capsule and phallus, gen. slide no. YC00024 6 L. wuzhishanensis Yin, sp. nov., paratype, female genitalia, gen. slide no. YC00023 6a L. wuzhishanensis Yin, sp. nov., enlargement of corpus bursae, gen. slide no. YC00023 7 L. jianfengensis Yin, Wang and Park, female genitalia, gen. slide no. YC00028 7a L. jianfengensis Yin, Wang and Park, enlargement of corpus bursae, gen. slide no. YC00028. Scale bars: $0.40 \mathrm{~mm}$.

Male genitalia (Fig. 4): Uncus broad at base, gradually narrowed to about $1 / 2$, apical half parallel bilaterally, apex rounded; gnathos obtriangular, with minute granules on apical half; tegumen with nearly trapezoid posterior margins fused with uncus, anterior 

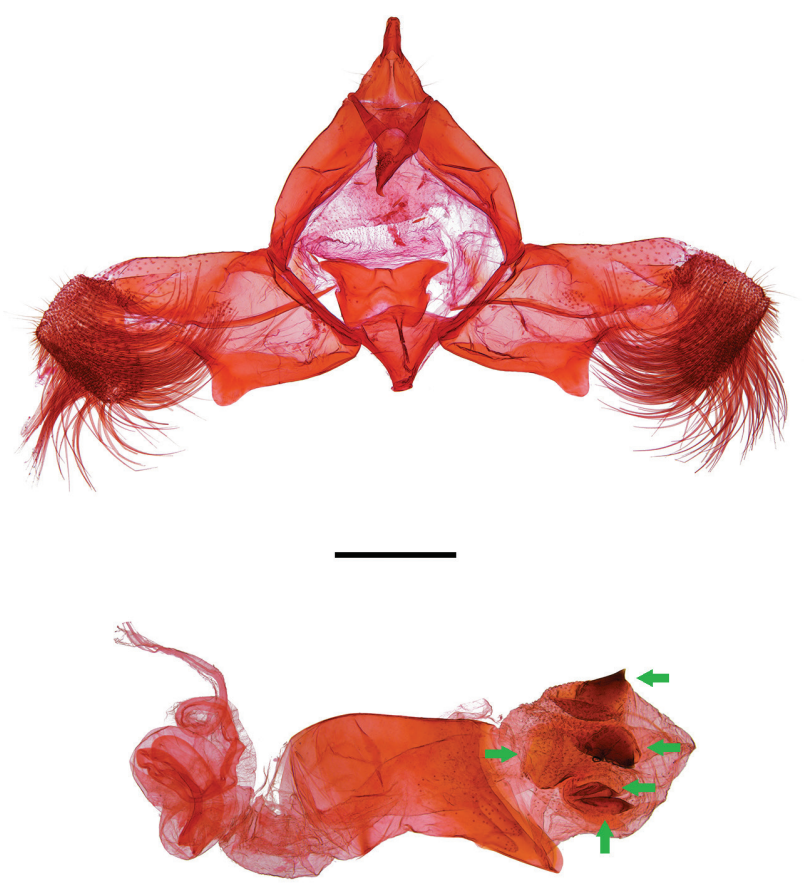

8

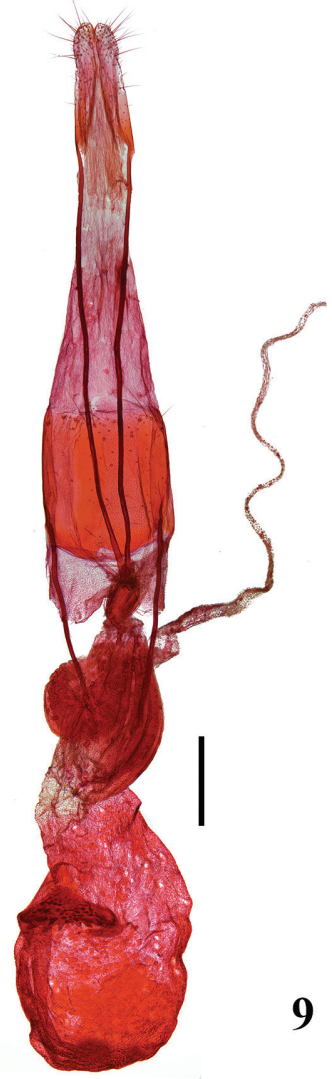

Figures 8, 9. Male and female genitalia of L. pallidiptera Yin, Wang and Park 8 male genital capsule and phallus, gen. slide no. YAH19075 9 female genitalia, gen. slide no. YAH18160. Scale bars: $0.40 \mathrm{~mm}$.

margin deeply emarginate; valva short and broad, sub-rectangular, heavily setose on triangular area apically; costa nearly straight; ventral margin triangularly concave inward beyond end of sacculus; sacculus narrow, slightly arched ventrally, distally with a triangular process directing downward; vinculum narrowly banded; saccus short, triangular, apex bluntly rounded; juxta obtrapezoidal, posterior margin straight, anterior margin concave mesially, lateral margins deeply emarginate at middle, forming two opposable triangular arms; phallus stout, distal $1 / 4$ protuberant, extending to a sharp point; vesica with six irregularly-shaped, plate-like cornuti, and one small cornutus (Fig. 4, arrows).

Female genitalia (Fig. 6a): Papillae anales setose, broadly rounded posteriorly; apophyses posteriores about 2.5 times length of apophyses anteriores; eighth sternite with posterior margin straight, setose on posterior half, darkly pigmented mesolaterally, paler mesolongitudinally; antrum wide, membranous; ductus bursae narrow and sclerotized posteriorly, elongate, with undulating internal sclerotization anteriorly, bearing inception of ductus seminalis on anterior part; ductus bursae subspherical, with a spiculate inner wall; signum a transverse, dentate band, with a posteriorly projecting semicircular lobe.

Remarks. Lasiochira wuzhishanensis Yin, sp. nov. is very similar in wing pattern to L. jianfengensis Yin, Wang and Park (Figs 1,2), but differs by having juxta with pos- 
terior margin straight (Fig. 4); phallus with seven cornuti (Fig. 4, arrows); and corpus bursae small, nearly spherical in shape (Fig. 6a). Lasiochira jianfengensis has juxta with posterior margin emarginate mesially (Fig. 5); phallus with six cornuti (Fig. 5, arrows); and corpus bursae ovate in shape and larger (Fig. 7a).

Biology. Unknown. Adults were attracted to white light in May.

Distribution. Known only from the type locality (China: Hainan province).

Etymology. The species epithet is derived from the name of the type locality, Wuzhishan National Nature Reserve.

\section{Lasiochira pallidiptera Yin, Wang \& Park, 2014}

Figs 3, 8, 9

Lasiochira pallidiptera Yin, Wang \& Park, 2014: 30 (Lasiochira; type locality: Mt. Godae-san, Yeoncheon-gun, Gyeongbuk province, Korea).

Material examined. $3 \hat{\partial} \widehat{\partial}, 1$ + ; China, Hubei province, Huanggang City, Yingshan County, Taohuachong Forest Park; alt. 700 m; 3059'04"N, 11556'15"E; 5 Jun. 2018;

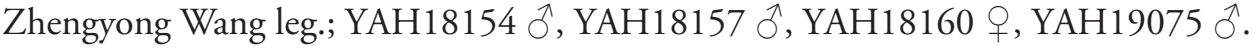

Remarks. This species is recorded from China for the first time.

Biology. Unknown. Adults were attracted to white light in June.

Distribution. China (Hubei province), Korea (Central).

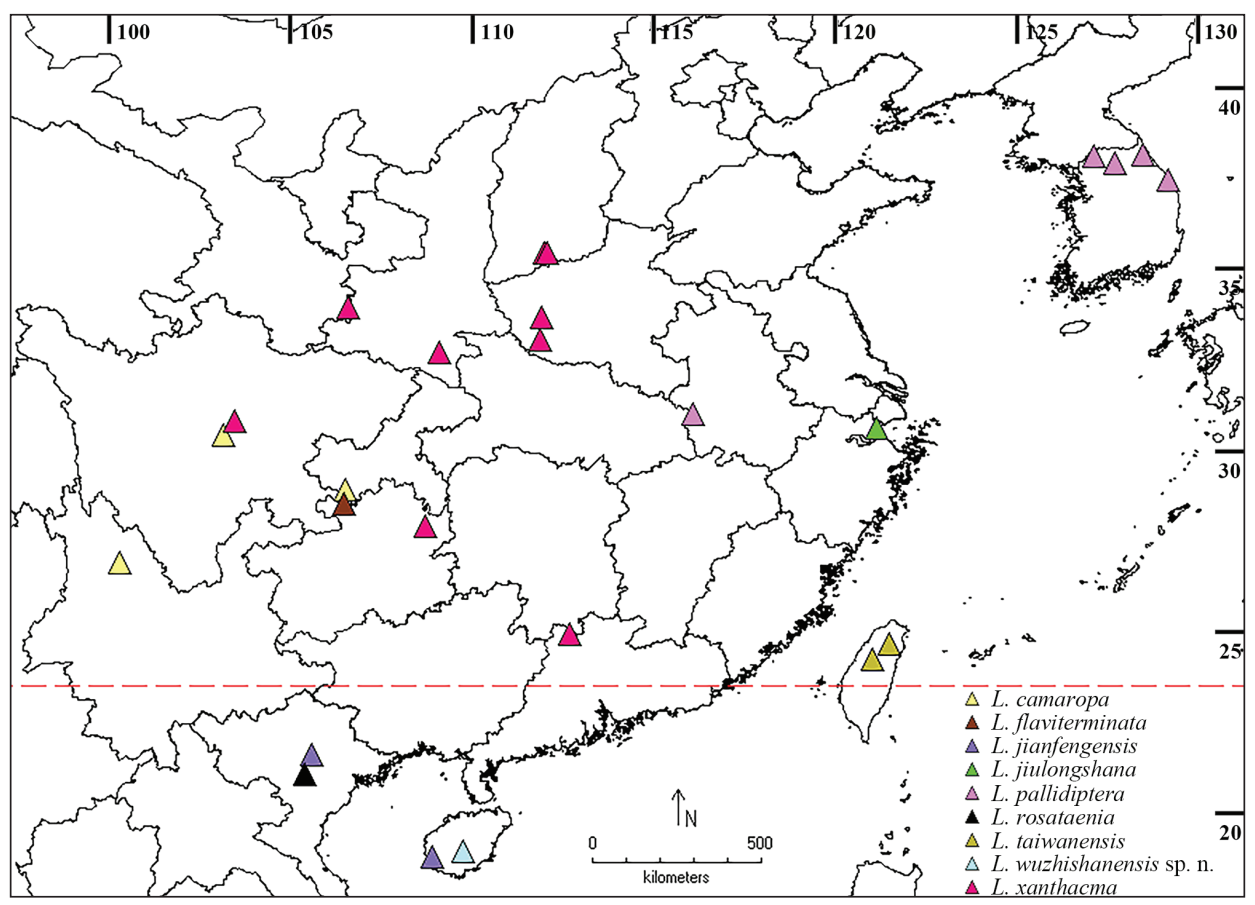

Figure 10. Distribution map of Lasiochira species. 


\section{Acknowledgements}

We want to thank the editors and the reviewers for reviewing this manuscript and giving a lot of constructive suggestions. This project was supported by the National Natural Science Foundation of China (No. 31760630, No. 31760629) and the Guizhou Provincial Department of Education Youth Science and Technology Talent Growth Project (黔教合KY字[2017]175, Guizhou Education KY word [2018]211).

\section{References}

Clarke JFG (1963) Catalogue of the Type Specimens of Microlepidoptera in the British Museum (Natural History) described by Edward Meyrick. Trustees of the British Museum (Natural History), London, England 4: 1-521.

Hijmans RJ, Guarino L, Rojas E (2011) DIVA-GIS, version 7.5. A geographical information system for the analysis of biodiversity data. http://www.diva-gis.org [accessed 23 December 2019]

Kristensen NP (2003) Skeleton and muscles: Adults. In: Kristensen NP (Ed.) Lepidoptera, Moths and Butterflies, 2 Morphology, physiology and development. Handbook of Zoology, De Gruyter, Berlin, New York 4(36): 39-131. https://doi.org/10.1515/9783110893724.39

Meyrick E (1913) Carposinidae, Heliodinidae, Glyphipterygidae. In: Wagner H (Ed.) Lepidopterorum Catalogus, Pars 13, W. Junk, Berlin 1-53.

Meyrick E (1931) Second contribution to our knowledge about the Pyralidae and Microlepidoptera of Kwanhsien. Bulletin de la Section Scientifique de l'Académie Roumaine 14: 59-75.

Meyrick E (1938) Materialien zu einer Mikrolepidopterenfauna des Yülingshanmassivs (Provinz Yünnan). Deutsche Entomologische Zeitschrift, Iris 52: 1-29.

Wang SX (2006) Oecophoridae of China (Insecta: Lepidoptera). Science Press, Beijing 258 pp. [15 col. pls.]

Yin AH, Wang SX, Park KT (2014) Review of the genus Lasiochira Meyrick, 1931 (Lepidoptera: Oecophoridae). Zootaxa 3802(1): 23-34. https://doi.org/10.11646/zootaxa.3802.1.2 